\title{
Content-based video copy detection based on motion vectors estimated using a lower frame rate
}

\author{
Kasım Taşdemir • A. Enis Çetin
}

Received: 20 October 2013 / Revised: 3 January 2014 / Accepted: 10 February 2014 / Published online: 17 March 2014

(C) Springer-Verlag London 2014

\begin{abstract}
We propose a motion vector-based video contentbased copy detection method. One of the signatures of a given video is motion vectors extracted from image sequences. However, when consecutive image frames are used, the resulting motion vectors are not descriptive enough because most vectors are either too small or they appear to scatter in all directions. We calculate motion vectors in a lower frame rate than the actual frame rate of the video to overcome this problem. As a result, we obtain large vectors and they represent a given video in a robust manner. We carry out experiments for various parameters and present the results.
\end{abstract}

Keywords Content-based copy detection $\cdot$ Similar video detection - Motion vectors - Sequence matching .

Video copy detection

\section{Introduction}

Detecting videos violating the copyright of the owner comes into question by growing broadcasting of digital video on different media. Content-based copy detection (CBCD) is an alternative way to watermarking approach to identify the ownership of video. In this approach, the video itself is considered as a watermark. Existing methods of video CBCD usually extract signatures, key frames, or fingerprints from

This work is published in part at IEEE ICPR Conference in Istanbul, 2010.

K. Taşdemir $(\varangle) \cdot$ A. E. Çetin

Department of Electrical and Electronics Engineering,

Bilkent University, 06800 Ankara, Turkey

e-mail: kasimtasdemir@gmail.com; tasdemir@ee.bilkent.edu.tr

A. E. Çetin

e-mail: cetin@bilkent.edu.tr the images of video stream and compare them with the database that contains the features of original videos $[2,6,13-$ 16]. Several spatial or temporal features of videos are considered as signatures of videos such as intensity of pixels, color histograms, and motion $[3,9]$. The main advantage of $\mathrm{CBCD}$ over watermarking is that signature extraction can be done even if the video is distributed over the Internet or other media because the unique signature is part of the video itself.

In CBCD algorithms, average color, color histogram, and motion are used as feature parameters or vectors. Each feature set has advantages over others. When a movie is recorded from a movie theater by a handheld camera, then its color map, fps, size and position change, and edges get soften. Color-based algorithms will have difficulties detecting the camera-recorded copy of an original movie because the information it depends on is significantly disturbed. However, motion in a copied video remains similar to the original video.

Motion information was considered as a weak parameter by other researchers [3]. In [12], it is shown that this is not true unless the motion vectors are extracted from consecutive frames in a video with a high capture rate. Most motion vectors are small or close to zero in a typical $25 \mathrm{~Hz}$ captured video and they may not contain any significant information. They also appear to scatter in all directions due to incorrect motion vector calculation because neighboring pixel values are close to each other in consecutive video frames. On the other hand, if we can detect the general motion trends in a video as representive of the video, we get a reliable feature set of parameters. In this article, we calculate motion vectors in a lower frame rate than the actual frame rate of the video. As a result, we obtain larger vectors compared to the motion vectors obtained at a higher rate and we experimentally show that they represent a given video in a robust manner. 


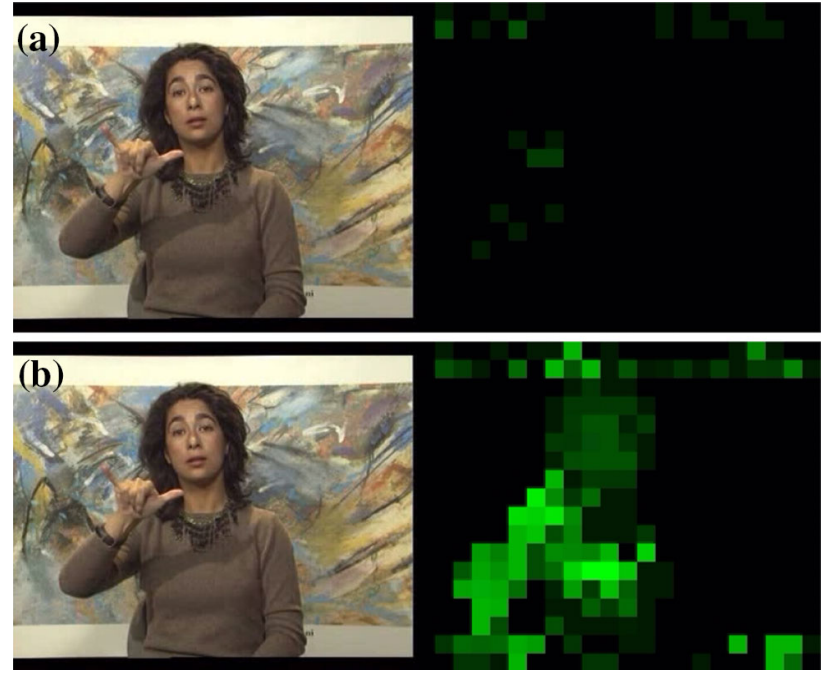

Fig. 1 Effect of lower fps in the motion vector estimation algorithm: a 151th frame and its corresponding MV pattern of video "silent." MVs are extracted using the next frame. The MV magnitudes are small. b 151 th frame of video "silent." MVs are extracted using every 5 th frame. The MV magnitudes are larger than (a)

\subsection{Motion vector (MV) extraction and feature sets}

In general, motion vectors are extracted using consecutive frames in many video analysis and coding methods. In order to capture the temporal behavior more efficiently, we use every $t$ th and $(t+n)$ th, $n>1$ frames instead of the traditional approach of using $t$ th and $(t+1)$ th frames. In our approach, we use every $t$ th and $(t+n)$ th frame for motion vector extraction. For example, human movements change slowly in a $25 \mathrm{fps}$ video. If two consecutive frames are used in motion vector extraction step, resulting motion vectors will have small values because of the high capture rate of the video. Furthermore, some of the image blocks (or macroblocks) inside the moving object may be incorrectly assumed as stationary or moving in an incorrect direction by the motion estimation algorithm because similar image blocks may exist inside the moving object. By computing the MVs using every $n$-th frame $(n>1)$, it is possible to get more descriptive motion vectors. In Fig. 1, instead of using two consecutive frames, we use $t$ th and $(t+5)$ th frames for MV computation, and as a result, MV displacements in the video will be high. As shown in Fig. 1, moving objects are clearly emphasized. We define the mean of the magnitudes of motion vectors (MMMV) of macroblocks of a given frame as follows:

$\operatorname{MMMV}(k)=\frac{1}{N} \sum_{i=0}^{N-1} r(k, i)$

where $r(k, i)$ is the motion vector magnitude of the macroblock in position $i$ of $k$ th frame and $N$ is the number of macroblocks in an image frame of the video. We also define
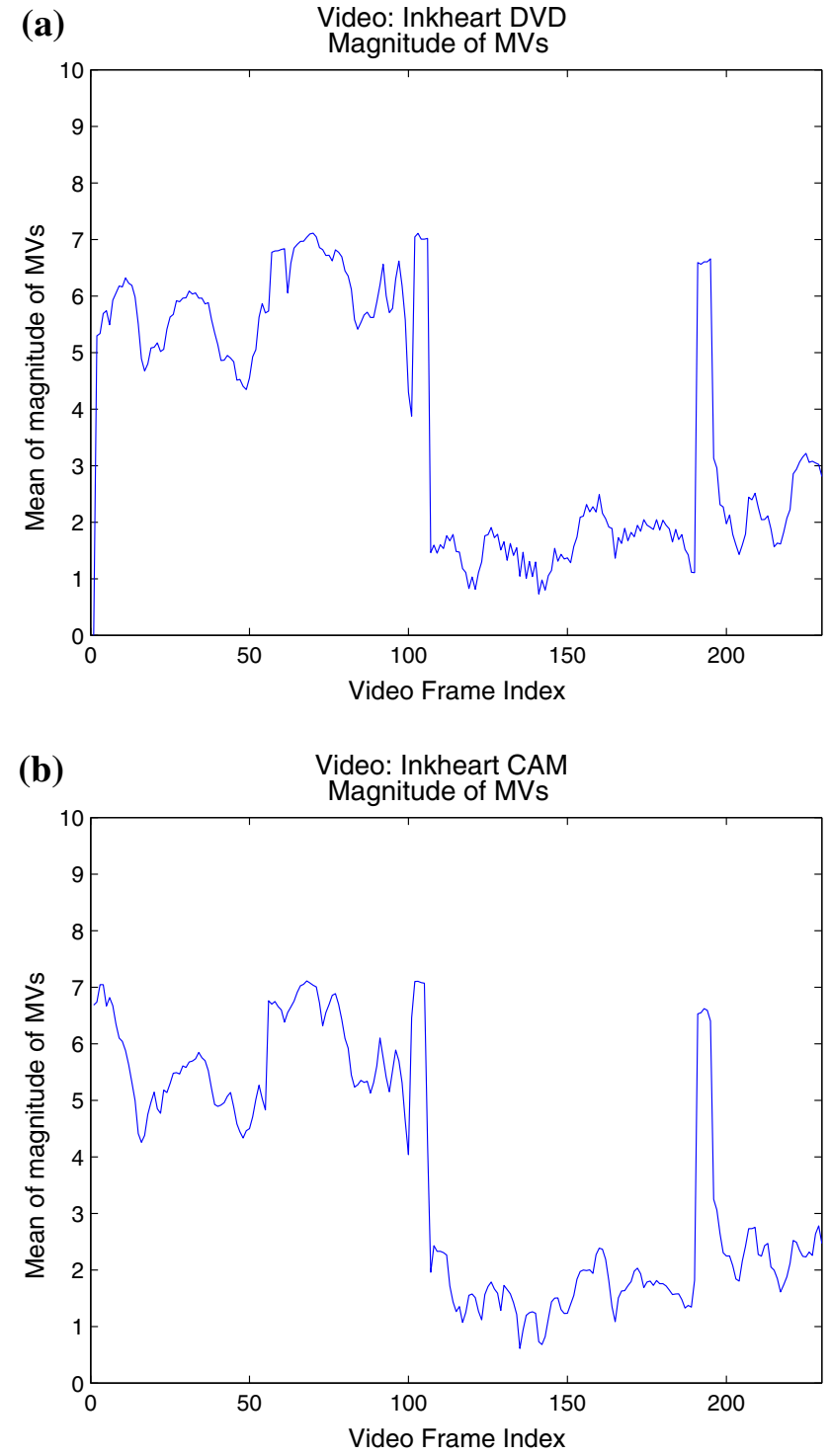

Fig. 2 Similarity of the MMMV plots of "Inkheart DVD" and "Inkheart CAM," (with $n=5$ )

the mean of the phase angles of motion vectors (MPMV) of macroblocks of a given frame as follows:

$\operatorname{MPMV}(k)=\frac{1}{N} \sum_{i=0}^{N-1} \theta(k, i)$,

where $\theta(k, i)$ is the motion vector angle of the macroblock in position $i$ of the $k$ th frame of the video and $N$ is the number of macroblocks. The angle $\theta$ is in radians and $\theta \in(-\pi, \pi)$. So, the range of MPMV is also in the same region: $\operatorname{MPMV}(\cdot) \in$ $(-\pi, \pi)$.

We use the discrete MMMV $(k)$ and $\operatorname{MPMV}(k)$ functions as the feature sets representing a given video. Example MMMV and MPMV plots are shown in Figs. 2 and 7, respectively. Storage requirement is low as both functions require a single 
real number for each frame $k$ of the video. It is possible to divide the image frames into subimages and extract MMMV and MPMV values for each subimage but we experimentally observe that a single value for a given frame is sufficient to characterize a video.

In the following subsection, we describe the method that we used for motion vector (MV) estimation in video.

\subsection{Motion vector extraction}

We extract motion vectors from image frames using the simple and efficient search (SES) algorithm [10] and use an exhaustive search (ES) [1] for block-matching. However, other motion vector estimation methods can be also used.

Block-matching is performed on the current frame $(t)$ and a previous frame $(t-u)$. The current frame is divided into square blocks of pixel size $N \times N$ called macroblocks (MB). Each block has a search area in the previous frame, which has the size $(2 W+N+1) \times(2 W+N+1)$ where $W$ is the amount of maximum vertical or horizontal displacement. Then, the best matching block is searched in the previous frame using the current block. The motion vector is defined as the $(x, y)$ which makes the mean absolute difference (MAD) minimum. The MAD is expressed as

$$
\begin{aligned}
\operatorname{MAD}(x, y)= & \frac{1}{N^{2}} \sum_{i=0}^{N-1} \sum_{j=0}^{N-1} \mid F_{\mathrm{c}}(k+i, l+i) \\
& -F_{\mathrm{p}}(k+x+i, l+y+j) \mid
\end{aligned}
$$

where $F_{\mathrm{c}}(\cdot, \cdot)$ and $F_{\mathrm{p}}(\cdot, \cdot)$ are pixel intensities of the current and the previous frames, respectively, $(k, l)$ is the horizontal and vertical coordinates of the upper left corner of the image block, and $(x, y)$ is displacement in pixels [10].

\subsection{Exhaustive search algorithm}

Another name of this algorithm is the Full Search algorithm. This calculates MAD for all possible locations in a given search window. As a result, it gives the best possible match and the highest PSNR among any block-matching algorithms [1]. This algorithm is straightforward to implement and gives the best results. The disadvantage of this algorithm is its high computational cost.

\subsection{A simple and efficient search algorithm}

This algorithm is a modified version of the three-step search (TSS) algorithm $[1,10]$. In the TSS algorithm, a block is searched in some reference points of locations in the previous frame instead of searching all possible locations. First, points in the center and eight points around the center are checked. If the minimum is at the lower right point, the search algorithm continues in the same manner with a smaller search
Table 1 Properties of original movies (with DVD extension) and the same movies recorded from a handheld camera (with CAM extensions)

\begin{tabular}{lll}
\hline Movie name & Frames per second (FPS) & Video size \\
\hline Desperaux DVD & 24 & $640 \times 272$ \\
Desperaux CAM & 25 & $608 \times 304$ \\
Inkheart DVD & 25 & $624 \times 352$ \\
Inkheart CAM & 25 & $704 \times 304$ \\
Mallcop DVD & 30 & $608 \times 320$ \\
Mallcop CAM & 24 & $720 \times 320$ \\
Spirit DVD & 24 & $640 \times 272$ \\
Spirit CAM & 25 & $656 \times 272$ \\
\hline
\end{tabular}

window. After applying it three times, the location that gives the minimum MAD is found. The motion vector is decided as a vector from the center to that point.

\section{Video copy detection using MMMV and MPMV}

Searching and comparing the movies violating the copyright issues with official movies may not be a challenging problem if we know that the copied movie has exactly the same digital data as the original. However, in most cases, unofficial movies are published with a small distortion or additions such as resizing, cropping, zooming in and out, adding a logo, changing the fps, and changing color. Most encountered reallife example is the distribution of handheld camera-recorded movies of new movies from the movie theater. Since this unofficially made copy is a completely new record, it loses some of the features of the original movie. For instance, colors will change both due to the projector illuminating the curtain and during the camera recording. Depending on the quality of the recording device, its view point, and its orientation, recorded movie may lose edges in frames or it may have different scale and perspective than the original movie. Color histogram-based CBCD comparison methods have the disadvantage that they depend on the distorted color information. However, the motion vectors do not change as much as color information. This section investigates the similarity of MMMV-MPMV data of original movies and their handheld camera versions. Table 1 shows the properties of the movies used in this section. Test videos have different size and fps. Videos with CAM extensions are copies obtained using a handheld camera. In Sect. 3, we present extensive comparisons using a video database. Although the original and handheld camera-recorded videos have different fps and size, they have similar MMMV plots as shown in Fig. 2. Original movie in Fig. 2a and its handheld camera-recorded version from a movie theater (Fig. 2b) show significant similarities. The MVs are computed with a frame difference of $n=5$. In order to obtain a value that gives informa- 
tion about how much two movies resemble each other, the absolute difference is calculated as distance, $D$. Differencing the two features directly is not a good solution because of two reasons. The first reason is that they may have different fps values. So, each index of the original video should be compared with its corresponding index of the candidate video in terms of real time. However, most of the indices do not correspond to the same time instant. After calculating the indices corresponding to the nearest time instant, we use a search window in order to compare it with also its neighbors.

The second reason is that the sizes of frames of the videos can be different. If frame sizes are different, motion vectors of videos will be also different. The video with a larger frame size will have larger motion vectors. The MMMV data of videos will be scaled version of each other. In order to solve this problem, we first normalize the MMMV and MPMV of the videos before making a comparison as follows:

$\overline{\operatorname{MMMV}}(t)=V(t)=\frac{\operatorname{MMMV}(t)-\mu_{\mathrm{MMMV}}}{\sigma_{\mathrm{MMMV}}}$

where $\mu_{\mathrm{MMMV}}$ is the mean and $\sigma_{\mathrm{MMMV}}$ is the standard deviation of the MMMV array, respectively.

The sum of absolute values of difference of normalized MMMV values of two videos $o$-original and $c$-copy are calculated as the distance $D(o, c)$ as follows:

$D(o, c)=\frac{1}{N} \sum_{t} \min _{|d| \leq W}\left|V_{\mathrm{o}}(t)-V_{\mathrm{c}}(t+d)\right|$

where $W$ is the search window width. We time align the videos manually and select $W$ as 2 frames because the fps of most commercial videos are between 20 and 30. In Eq. 5, $N$ is the number of frames in the movie $\mathrm{MMMV}_{\mathrm{o}}$. If the original and the candidate video have different fps, then their frame indices corresponding to the same time instance should be calculated first. So, instead of comparing corresponding frame indexes, the aim is to compare image frames corresponding to the same time instant.

We define another measure of the distance between two video clips based on estimating the $V_{\mathrm{c}}(t)$ sequence of the video clip $c$ using the $V_{\mathrm{o}}(t)$ of the video clip $o$ as follows:

$D(o, c)=\frac{1}{N} \sum_{t}\left|V_{\mathrm{o}}(t)-\sum_{k=-L}^{L} w_{k, t} V_{\mathrm{c}}(t-k)\right|$

where $w_{k, t}$ are the weights of the $2 L+1$ order linear estimator. The weights are adaptively updated using the well-known LMS algorithm:

$w_{k+1, t}=w_{k, t}+\lambda e(t) V_{\mathrm{c}}(t)$
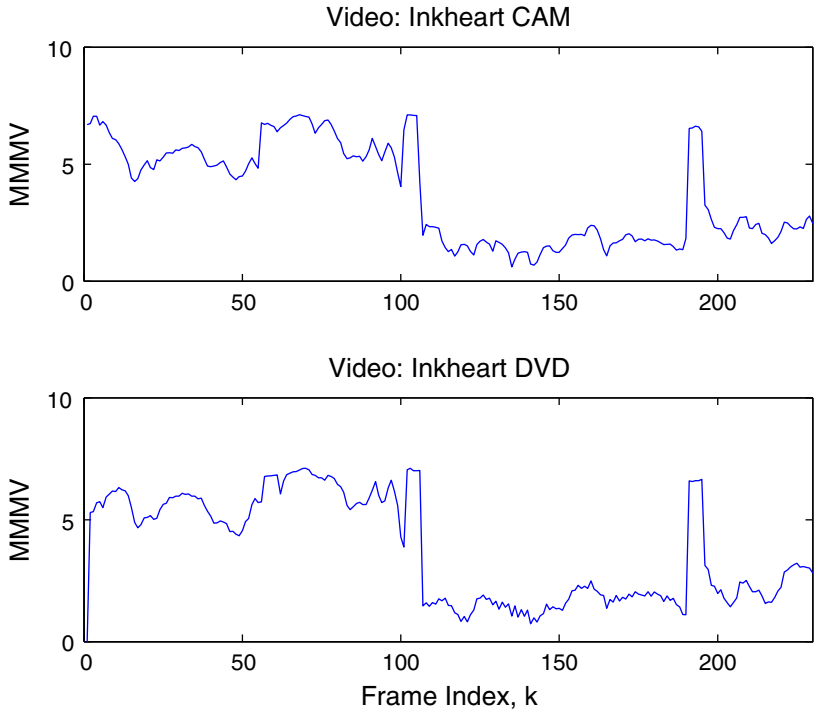

Fig. 3 MMMV plots of videos "Inkheart DVD" and "Inkheart CAM" videos. The distance between the MMMV plots, $D(o, c)=0.35$
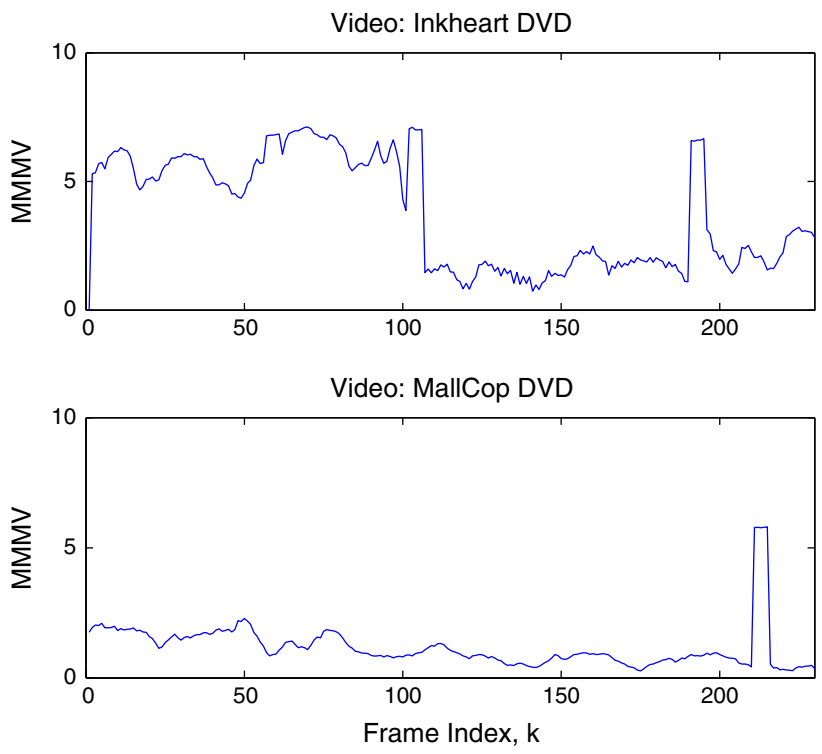

Fig. 4 MMMV plots of "Inkheart DVD" and "Mallcop.CAM" videos. The distance between the MMMV plots, $D(o, c)=2.91$

where $\lambda$ is the adaptation constant and

$e(t)=V_{\mathrm{o}}(t)-\sum_{k=-L}^{L} w_{k, t} V_{\mathrm{c}}(t-k)$

is the estimation error at frame $t$. The parameter $\lambda$ can be selected as in the normalized LMS algorithm.

The distance $D$ of a video of an original movie Inkheart and the same video recorded with a handheld camera is shown in Fig. 3. The last plot shows the absolute of frame-by-frame $\overline{\mathrm{MMMV}}$ difference. Since the $\overline{\mathrm{MMMV}}$ plot of the two videos is similar, their average of absolute difference value is small, 
Table 2 Average $\mathrm{MMMV}_{N}$ distance of test videos

\begin{tabular}{lllll}
\hline Distance & V1 CAM & V2 CAM & V3 CAM & V4 CAM \\
\hline V1 DVD & 0.44 & 1.23 & 0.9 & 0.86 \\
V2 DVD & 1.2 & 0.08 & 0.68 & 0.74 \\
V3 DVD & 0.85 & 0.54 & 0.18 & 0.75 \\
V4 DVD & 1.06 & 0.76 & 0.67 & 0.29 \\
\hline
\end{tabular}

Italicize values the distance of original and its copy. V1-V4 stands for the names of the test videos "Desperaux," "Inkheart," "Mallcop," "Spirit"
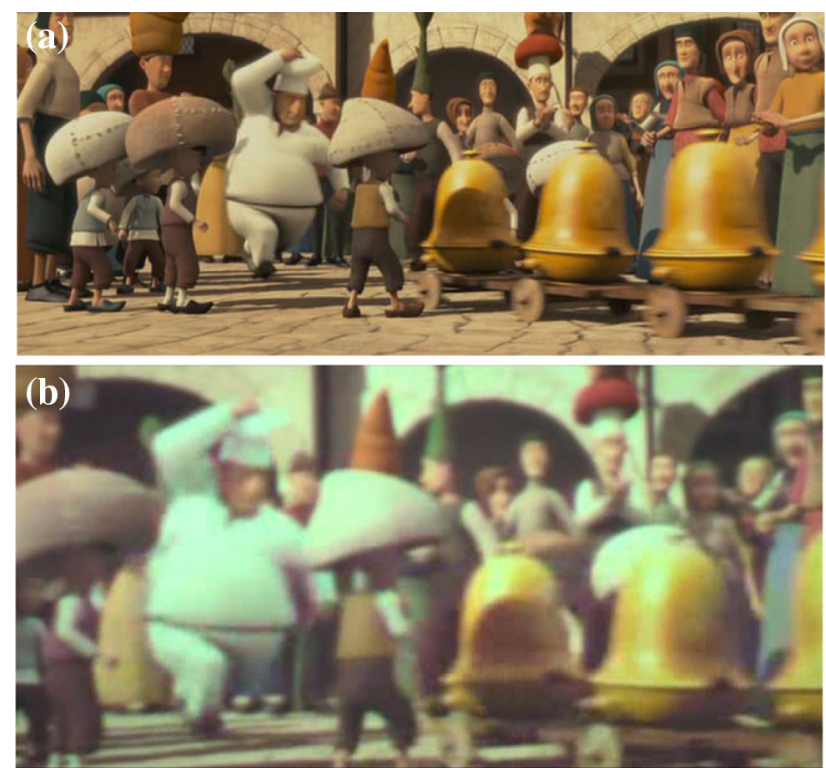

Fig. 5 The same frames of videos "Desparaux.DVD" and "Desparaux.CAM," a the original movie frame and $\mathbf{b}$ the same frame for the video recorded by a handheld camera. It is highly distorted

0.35. However, the distance of two different videos are not small as shown in Fig. 4. Since the two different movies have different camera motions and object movements, their $\overline{\mathrm{MMMV}}$ plots are not similar, $D(o, c)=2.91$. However, distance of original video $o$ and handheld camera-recorded video $c$ is $0.35, D(o, c)=0.35$.

Comparison of distances of 8 test videos are listed in Table 2. Rows of Table 2 are original videos, and columns are handheld camera-recorded versions. The diagonal elements of Table 2 are a measure of similarity of the original and copy of the video. Diagonal elements are expected to be smallest value in a given row because a video should be similar to its copy and different from the others. Diagonal elements are the smallest values, which mean that the original videos are most similar to their camcorder copy in terms of $\mathrm{MMMV}_{N}$. Although the camera recordings of video "Desperaux CAM" is at a very low quality and it has significant morphological distortions, it successfully paired with its original version. Sample screen shots of same frames of videos of "Desperaux CAM" and "Desperaux DVD" are shown in Fig. 6. Side
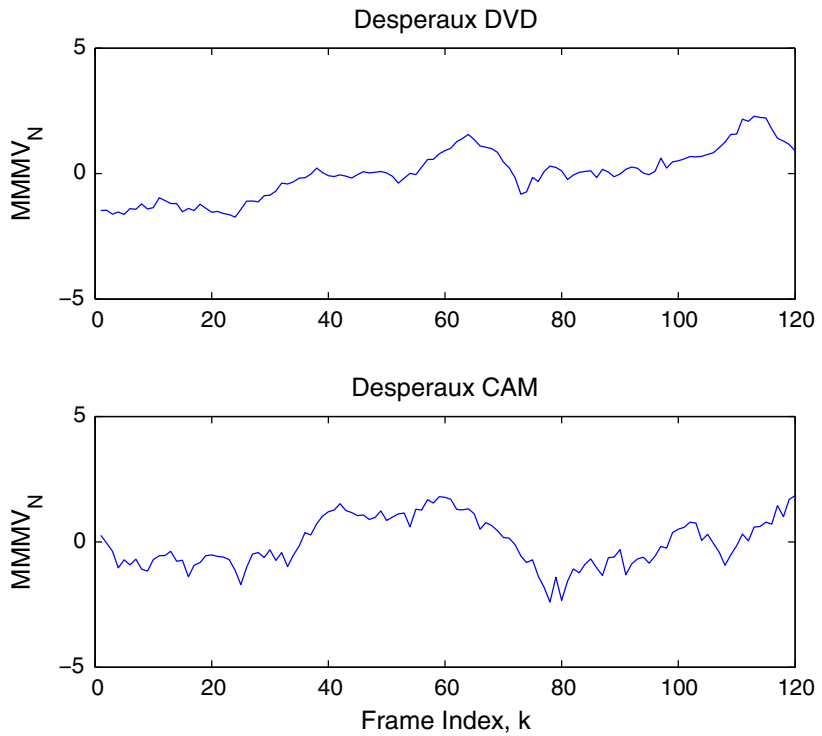

Fig. $6 \overline{M M M V}$ plots of "Desparaux.DVD" and "Desparaux.CAM" video clips. The distance between the MMMV plots, $D(o, c)=0.44$
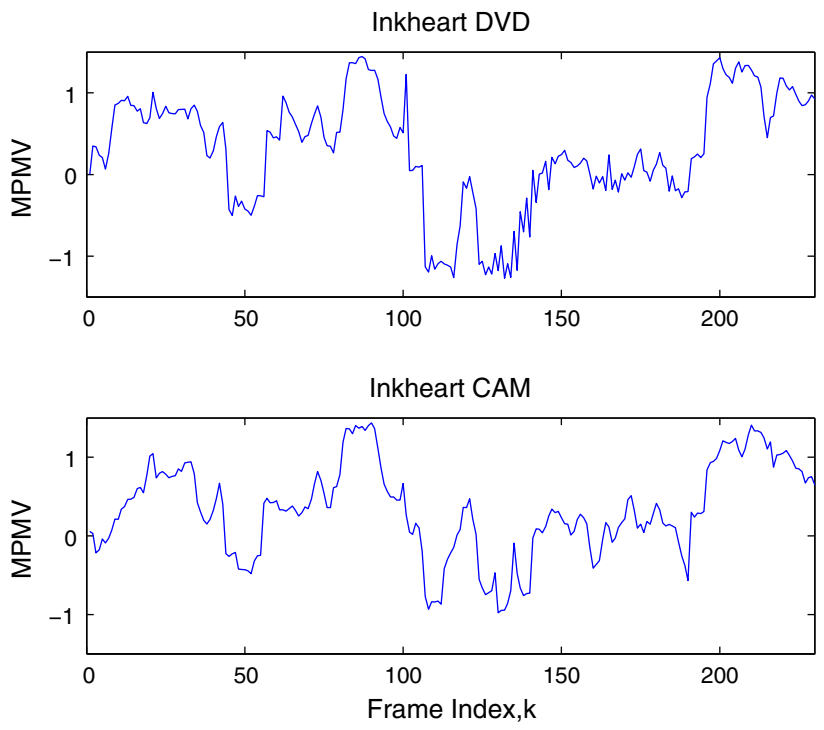

Fig. 7 The MPMV plots of "Inkheart DVD" and "Inkheart CAM" video clips. The distance between the MPMV plots, $D(o, c)=0.22$

portions of the video is lost because of zoom in of the handheld camera, and camera focus is not adjusted so it is very blurred. $\overline{\text { MMMV }}$ plot and the distance plot of "Desperaux DVD" and "Desperaux CAM" are shown in Fig. 5.

As discussed above, angle information of motion vectors can be also used for video copy detection. The MPMV plots of "Inkheart DVD" and "Inkheart CAM" are shown in Fig. 7. The original video and the recorded video have very similar MPMV plots. Comparison results of test videos are listed in Table 3. Diagonal elements of the Table 3 are the smallest elements in a given row in Table 3. The distance between the original video and the corresponding copy pair is the 
Table 3 Average distance $D$ of $\overline{\text { MPMV }}$ data of test videos

\begin{tabular}{lllll}
\hline Distance & V1 CAM & V2 CAM & V3 CAM & V4 CAM \\
\hline V1 DVD & 0.29 & 0.96 & 0.7 & 0.74 \\
V2 DVD & 1.03 & 0.15 & 0.85 & 0.86 \\
V3 DVD & 0.98 & 0.87 & 0.4 & 0.74 \\
V4 DVD & 0.62 & 0.75 & 0.59 & 0.24 \\
\hline
\end{tabular}

Italicize values show the distance between the original and its copy. V1-V4 stands for the names of the test videos "Desperaux," "Inkheart," "Mallcop," "Spirit"

Table 4 Video transformations

\begin{tabular}{ll}
\hline Transformation name & Transformation type \\
\hline T1 & A pattern inserted \\
T2 & Crop $10 \%$ with black window \\
T3 & Contrast increased by $25 \%$ \\
T4 & Contrast decreased by $25 \%$ \\
T5 & Zoom 1.2 \\
T6 & Zoom 0.8 with black window \\
T7 & Letterbox \\
T8 & Gaussian noise, $\mu=0, \sigma=0.001$ \\
\hline
\end{tabular}

smallest. So, $\overline{\text { MPMV }}$ data of similar videos are found to be the most similar data among test videos.

As discussed above, MMMV or MPMV information can be used as a feature of the video. Comparison results show that they can be used for the detection of artificially or manually modified versions of original videos. Each has superior sides. As it is shown in Table 3, phase informa- tion is more resistant to loss of some information and significant deformations in the video. Even magnitude data of the videos were not enough to detect the "Desperaux DVD" and "Desperaux CAM" as similar videos, phase data gave correct matching. On the other hand, MPMV is not rotation invariant but MMMV is rotation invariant. Therefore, both features should be used at the same time.

\section{Experimental results}

A video database is available in Ref. [4]. Original videos in this database are compared with the transformed versions of the same videos (Fig. 8). There are 47 original videos taken from Ref. [4]. Duration of the videos is 30 s. Each video has eight different transforms. The list of transformations is given in Table 4 . As a result, there are a total of $47 \times 9=423$ videos in the database. For each parameter set, 1,457 comparisons are performed.

Original videos are compared with test videos in the database and its eight transformations. For each test, the list of distance between the compared videos is calculated using Eq. 5 for different parameters or data types such as MMMV and MPMV.

The performance of each test is plotted using its receiver operating characteristics (ROC) curve. The ROC curve is a plot of false-positive rate $F_{\mathrm{pr}}$ and false-negative rate $F_{\mathrm{nr}}$, or true positive rate $T_{\mathrm{pr}}$. Let $F_{\mathrm{p}}, F_{\mathrm{n}}$ and $T_{\mathrm{p}}$ the number of false positives (clips that matched with a different video), false negative (clips that should match, but did not), and true positive (hit; clips that matched correctly in the positive set).
Fig. 8 Transformations: a original frame, $\mathbf{b}$ a pattern is inserted, c crop $10 \%$ with a black frame, $\mathbf{d}$ contrast increased by $25 \%$, e contrast decreased by $25 \%$, f zoom by 1.2 , $\mathrm{g}$ zoom by 0.8 with in the black window, h letterbox, $\mathbf{i}$ additive Gaussian noise with $\mu=0$ and $\sigma=0.001$
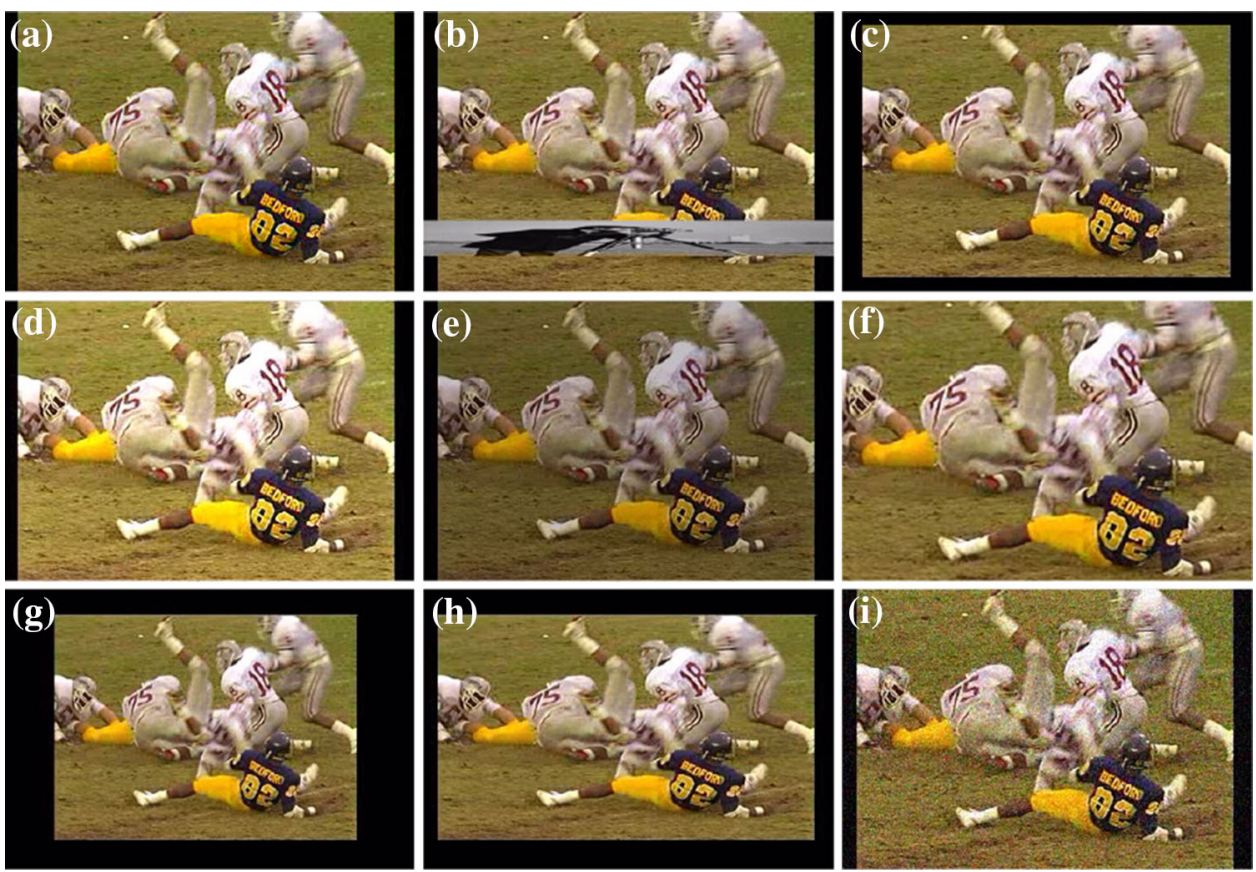


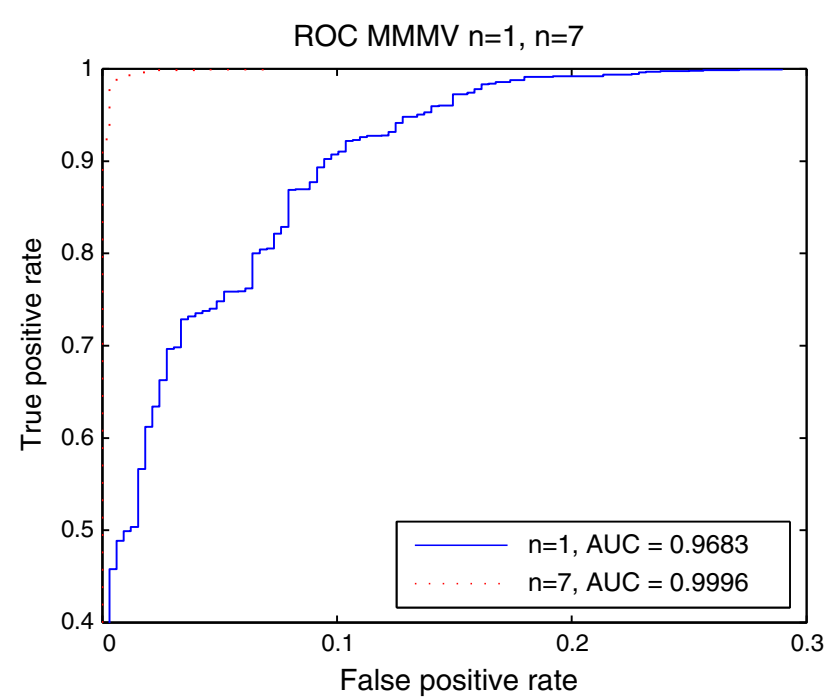

Fig. 9 Effect of increasing the frame distance $n=1$ to $n=7$ on MMMV ROC curves

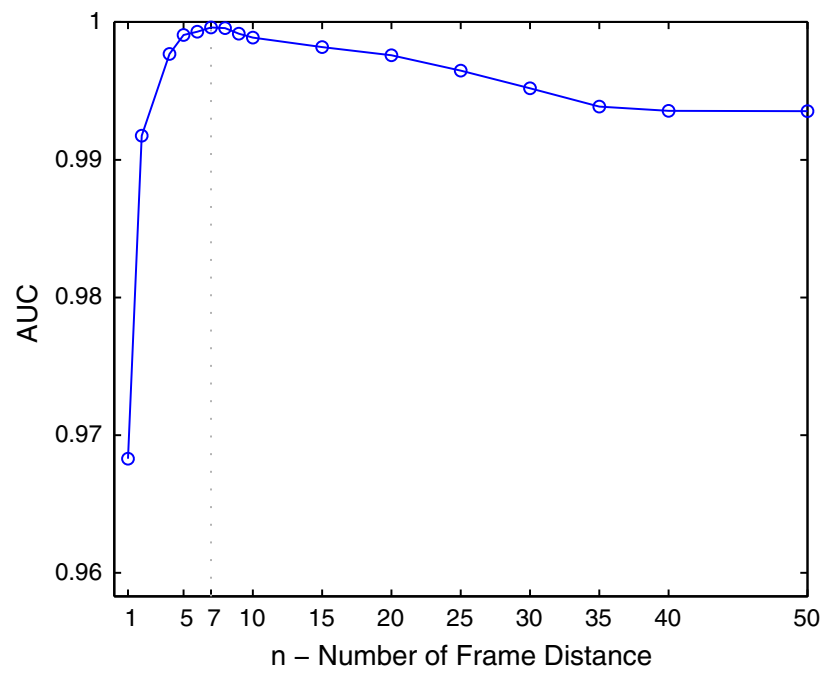

Fig. 10 Effect of varying $n$ on AUC of MMMV ROC ( $F_{\text {pr }}$ vs. $\left.T_{\text {pr }}\right)$ curves. The best result is obtained when $n=7$

False-positive, false-negative rates, and true-positive rate are defined as

$$
F_{\mathrm{pr}}(\tau)=\frac{F_{\mathrm{p}}}{N_{\mathrm{p}}}, F_{\mathrm{nr}}(\tau)=\frac{F_{\mathrm{n}}}{N_{\mathrm{n}}}, T_{\mathrm{pr}}=(\tau)=\frac{T_{\mathrm{p}}}{N_{\mathrm{p}}}
$$

where $N_{\mathrm{p}}$ and $N_{\mathrm{n}}$ are the number of maximum possible falsepositive and false-negative detections. Threshold is $\tau$ and its value is varied from 0 to its maximum value with an increment of $1 \%$.

Effect of increasing the frame-skipping parameter $n$ from 1 to 7 in motion vector extraction step is shown in Fig. 9 . We can obtain more descriptive features of videos based on motion vectors if we use every 7 th frame instead of the current and the next frame in motion estimation step. As it is shown in Fig. 10, there is a dramatic increase in detection ratio with

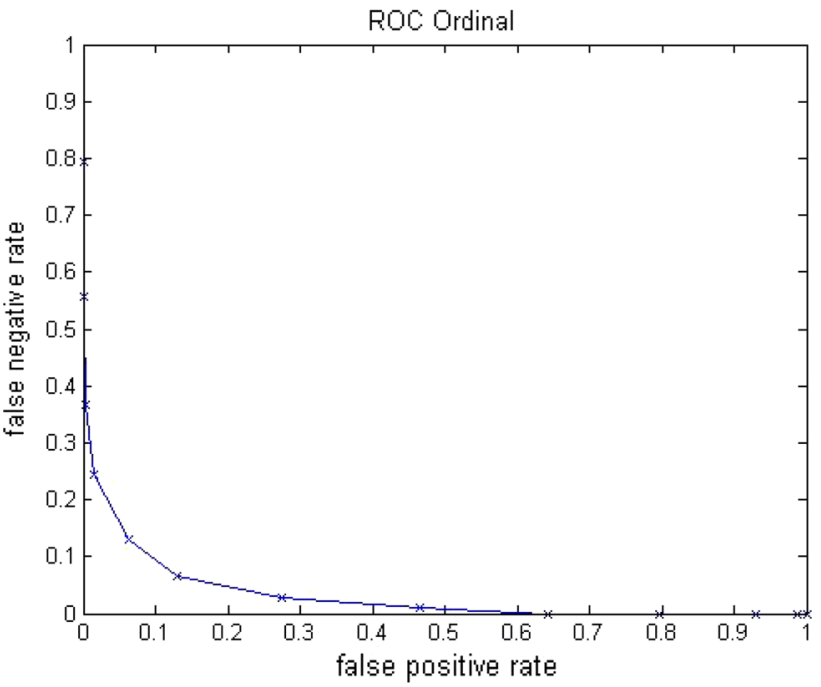

Fig. 11 The ROC curve $\left(F_{\mathrm{nr}}\right.$ vs. $\left.F_{\mathrm{pr}}\right)$ of the ordinal signature. In this video database, the MMMV and the MPMV signatures have better performance than the ordinal signature when the frame difference parameter $n=5$

increasing $n$ and we get the best result when $n=7$ where the area under curve (AUC) is 0.9996.

In Ref. [3], it is stated that ordinal signature introduced in Ref. [11] outperforms the Motion Signature. This is true when the motion vectors are extracted using the current and the next frame $(n=1)$. On the other hand, if motion vectors of videos are extracted using every 5 th frame, motion vectorbased MMMV plot is closer to the ideal case than the ROC curve of ordinal signature as shown in Fig. 11. The area under the ROC curve ( $F_{\mathrm{nr}}$ vs. $F_{\mathrm{pr}}$ ) of ordinal signature is 0.0311 , which is higher than the area under the ROC curves of both MMMV and the MPMV signatures.

As pointed above, the ROC curves of the proposed MMMV and MPMV methods are very close to each other as shown in Fig. 12. It is experimentally shown that the MMMV and the MPMV are good descriptive features for videos. In this database, the best results are obtained with the frame difference parameter $n=7$ as it is shown in Fig. 10.

\subsection{Number of feature parameters per frame}

Extracted features are stored in a database. The size of the database is important for practical reasons. Therefore, the number of features extracted for each frame is another important criteria for $\mathrm{CBCD}$ algorithms. Table 5 summarizes the feature per frame (FPF) values of several algorithms. The FPF values of algorithms except MMMV and MPMV are taken from Ref. [9].

Table 5 shows that MMMV and MPMV algorithms consume less space for signatures than the other algorithms except the method called "Temporal" [9]. 


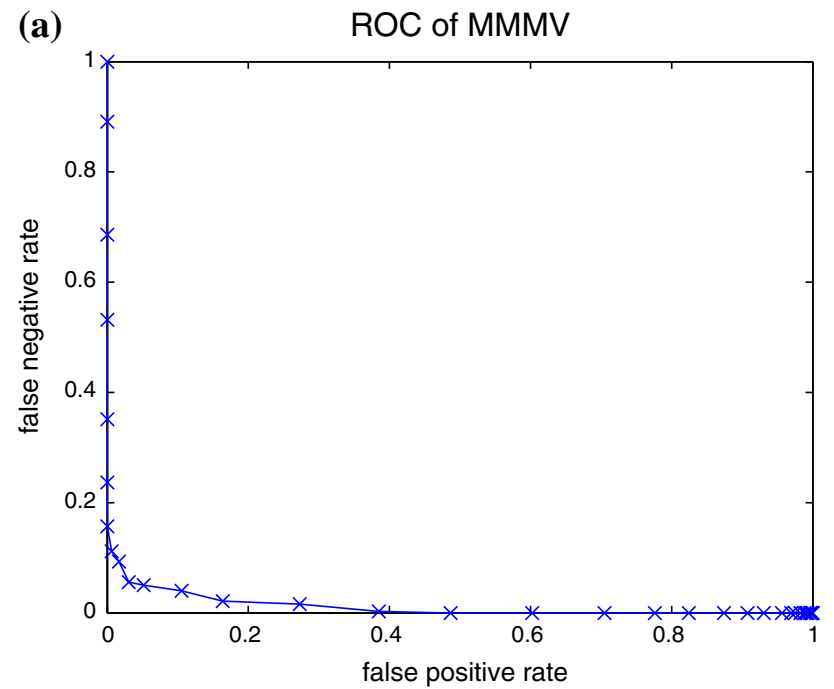

(b) ROC of MPMV

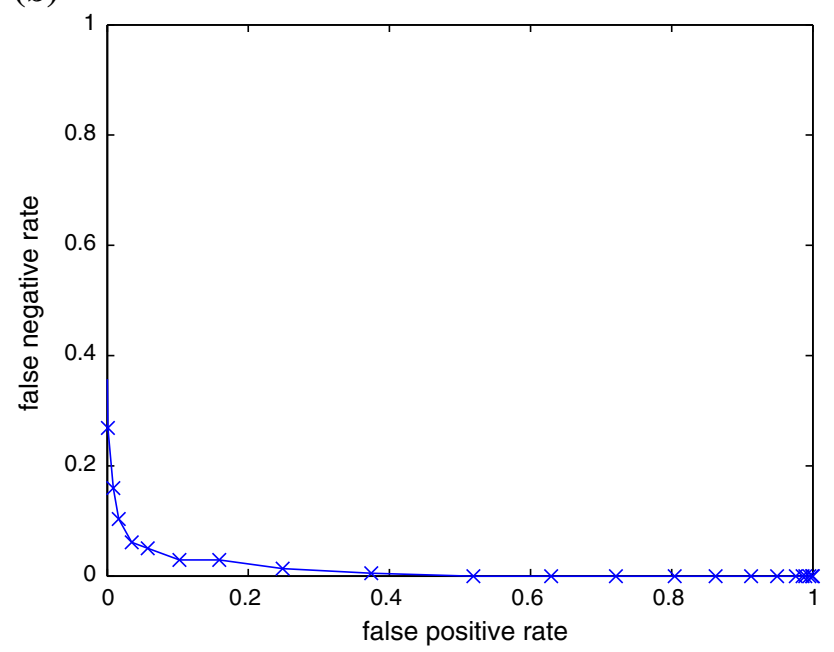

Fig. 12 The ROC curves of proposed methods when the frame difference parameter $n=5$. a MMMV and b MPMV

Table 5 Sizes of feature spaces

\begin{tabular}{ll}
\hline Technique & Features per frame \\
\hline ViCopT [8] & 7 \\
AJ [5] & 4.8 \\
STIP [7] & 73 \\
Temporal [9] & 0.09 \\
Ordinal Meas. [3] & 9 \\
MMMV & 1 \\
MPMV & 1 \\
\hline
\end{tabular}

\section{Conclusions}

In this article, we experimentally show that motion vectors are substantial signatures of videos as long as they are extracted in lower frame rates. Therefore, motion vectors can be used in CBCD algorithms. Primary aim of this article is to show that the motion vectors are good feature candidates (if $n=7$ ) for CBVCD algorithms rather than providing a complete CBVCD algorithm. A complete CBVCD algorithm would consist of database search algorithm. However, we manually align two videos before comparison.

Videos that have higher motion content give more reliable results and the videos having intensive motion activity are easier to distinguish when the neighboring image frames are used. However, videos containing slow-moving objects have very little motion vectors, and the vectors may appear to be random when the current and the next frame are used for motion vector computation.

In order to obtain reliable signature vectors for all videos motion vectors of the current, the next $n$th frame $(n>1)$ is used in motion vector estimation algorithms. Resulting motion vectors provide a reliable representation for all types of videos. Magnitude and phase angles of motion vectors are used separately as feature parameters of a given video. It is experimentally shown that both the magnitude and the phase of vectors can be considered as unique signatures of the video. The proposed motion-based feature parameters are resistant to illumination and color changes in video.

Motion vectors do not change significantly up to a level of resizing, croppingm, and blurring of the video. Most video copy detection methods are not robust to cropping. The MPMV feature is a robust feature in action videos because the moving objects are cropped in video as they are the information bearing part of a typical video and the direction of the object is the same in both the original and the cropped copy. If the recorded video is in low quality, then phase information is less affected than the magnitude information of the frames. However, MPMV is not rotation invariant but MMMV is rotation invariant. Therefore, it is better to use both MMMV and MPMV at the same time.

Another important comparison criterion of the $\mathrm{CBCD}$ algorithms in terms of the practical results is the size of the feature set in a database. The MMMV and the MPMV information do not occupy much space in the database as other methods. They both occupy one byte (one feature) per frame in the database.

\section{References}

1. Chan, E., Panchanathan, S.: Review of block matching based motion estimation algorithms for video compression. In: Electrical and Computer Engineering, Canadian Conference on, vol. 1, pp. 151-154 (1993). doi:10.1109/CCECE.1993.332213

2. Hampapur, A., Bolle, R.: Comparison of distance measures for video copy detection. In: Multimedia and Expo. ICME 2001. IEEE International Conference on, pp. 737-740 (2001)

3. Hampapur, A., Hyun, K., Bolle, R.M.: Comparison of sequence matching techniques for video copy detection. In: Yeung, M.M., Li, C.S., Lienhart, R.W. (eds.) Storage and Retrieval for Media 
Databases 2002, vol. 4676, pp. 194-201. SPIE(2001). doi:10.1117/ 12.451091, http://link.aip.org/link/?PSI/4676/194/1

4. Joly, A.: Internet archive movie database (2009). http://www. archive.org. [Online http://www.worldscinet.com/ijprai/ijprai. shtml; Accessed 10 Aug 2009]

5. Joly, A., Buisson, O., Frelicot, C.: Content-based copy retrieval using distortion-based probabilistic similarity search. Multimed. IEEE Trans. 9(2), 293-306 (2007). doi:10.1109/TMM.2006. 886278

6. Kobla, V., Doermann, D., Lin, K.I.D., Faloutsos, C.: Compressed domain video indexing techniques using DCT and motion vector information in mpeg video. In: Proceedings of the SPIE Conference on Storage and Retrieval for Still Image and Video Databases V, pp. 200-211 (1997)

7. Laptev, I., Lindeberg, T.: Space-time interest points. In: IN ICCV, pp. 432-439 (2003)

8. Law-To, J., Buisson, O., Gouet-Brunet, V., Boujemaa, N.: Robust voting algorithm based on labels of behavior for video copy detection. In: MULTIMEDIA '06: Proceedings of the 14th Annual ACM International Conference on Multimedia, pp. 835-844. ACM, New York, NY, USA (2006). doi:10.1145/1180639.1180826

9. Law-To, J., Chen, L., Joly, A., Laptev, I., Buisson, O., GouetBrunet, V., Boujemaa, N., Stentiford, F.: Video copy detection: a comparative study. In: CIVR '07: Proceedings of the 6th ACM International Conference on Image and Video Retrieval, pp. 371378. ACM, New York, NY, USA (2007). doi:10.1145/1282280. 1282336
10. Lu, J., Liou, M.: A simple and efficient search algorithm for blockmatching motion estimation. Circuits Syst. Video Technol. IEEE Trans. 7(2), 429-433 (1997). doi:10.1109/76.564122

11. Mohan, R.: Video sequence matching. In: Acoustics, Speech and Signal Processing. Proceedings of the 1998 IEEE International Conference on, vol. 6, pp. 3697-3700 (1998). doi:10.1109/ ICASSP.1998.679686

12. Tasdemir, K., Cetin, A.: Motion vector based features for content based video copy detection. In: Pattern Recognition (ICPR), 2010 20th International Conference on, pp. 3134-3137 (2010). doi:10. 1109/ICPR.2010.767.

13. Teodosio, L., Bender, W.: Salient video stills: content and context preserved. In: MULTIMEDIA '93: Proceedings of the First ACM International Conference on Multimedia, pp. 39-46. ACM, New York, NY, USA (1993). doi:10.1145/166266.166270

14. Tonomura, Y., Abe, S.: Content oriented visual interface using video icons for visual database systems. In: Visual Languages, IEEE Workshop on, pp. 68-73 (1989). doi:10.1109/WVL.1989. 77044

15. Yeung, M.M.Y.: Analysis, Modeling and Representation of Digital Video. Ph.D. Thesis, Princeton University, Princeton, NJ (1996)

16. Zhang, H., Kankanhalli, A., Smoliar, S.W.: Automatic partitioning of full-motion video. Multimed. Syst. 1(1), 10-28 (1993). doi:10.1007/BF01210504 